\section{Secondary hypoxic ischemia alters neurobehavioral outcomes, neuroinflammation, and oxidative stress in mice exposed to controlled cortical impact}

\author{
Se-Kwang Oh${ }^{1}$, Hyun-Jeong Park ${ }^{2}$, Gyeong-Gyu Yu², Seong-Hae Jeong ${ }^{3}$, \\ Suk-Woo Lee', Hoon Kim ${ }^{2,4}$ \\ 'Department of Emergency Medicine, Chungnam National University College of Medicine, Daejeon, Korea \\ ${ }^{2}$ Department of Emergency Medicine, Chungbuk National University College of Medicine, Cheongju, Korea \\ ${ }^{3}$ Department of Neurology, Chungnam National University College of Medicine, Daejeon, Korea \\ ${ }^{4}$ Department of Emergency Medicine, Chungbuk National University Hospital, Cheongju, Korea
}

Objective Hypoxic ischemia (HI) is a secondary insult that can cause fatal neurologic outcomes after traumatic brain injury ( $\mathrm{TBI})$, ranging from mild cognitive deficits to persistent vegetative states. We here aimed to unravel the underlying pathological mechanisms of $\mathrm{HI}$ injury in a TBI mouse model.

Methods Neurobehavior, neuroinflammation, and oxidative stress were assessed in a mouse model of controlled cortical impact (CCl) injury followed by HI. Mice underwent $\mathrm{CCl}$ alone, $\mathrm{CCl}$ followed by $\mathrm{HI}, \mathrm{HI}$ alone, or sham operation. $\mathrm{HI}$ was induced by one-vessel carotid ligation with 1 hour of 8\% oxygen in nitrogen. Learning and memory were assessed using the novel object recognition test, contextual and cued fear conditioning, and Barnes maze test. Brain cytokine production and oxidative stress-related components were measured.

Results Compared to TBI-only animals, TBI followed by HI mice exhibited significantly poorer survival and health scores, spatial learning and memory in the Barnes maze test, discrimination memory in the novel object recognition test, and fear memory following contextual and cued fear conditioning. Malondialdehyde levels were significantly lower, whereas glutathione peroxidase activity was significantly higher in TBI followed by HI mice compared to TBI-only and sham counterparts, respectively. Interleukin-6 levels were significantly higher in TBI followed by HI mice compared to both TBI-only and sham animals.

Conclusion Post-traumatic $\mathrm{HI}$ aggravated deficits in spatial, fear, and discrimination memory in an experimental TBI mouse model. Our results suggest that increased neuroinflammation and oxidative stress contribute to $\mathrm{HI}$-induced neurobehavioral impairments after TBI.

Keywords Traumatic brain injuries; Hypoxia; Inflammation; Behavior; Oxidative stress
elSSN: 2383-4625

Received: 7 September 2020

Revised: 14 January 2021

Accepted: 4 February 2021

Correspondence to: Hoon Kim Department of Emergency Medicine, Chungbuk National University College of Medicine, 1 Chungdae-ro, Seowongu, Cheongju 28644, Korea

E-mail: nichekh2000@chungbuk.ac.kr ORCID

https://orcid.org/0000-0002-2133-0311

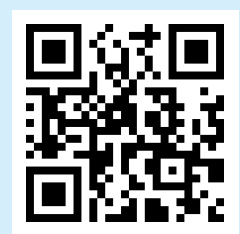

How to cite this article:

Oh SK, Park HJ, Yu GG, Jeong SH, Lee SW, Kim H. Secondary hypoxic ischemia alters neurobehavioral outcomes, neuroinflammation, and oxidative stress in mice exposed to controlled cortical impact. Clin Exp Emerg Med 2021;8(3):216-228. https://doi.org/10.15441/ceem.20.124

This is an Open Access article distributed under the terms of the Creative Commons Attribution Non-Commercial License (https:// creativecommons.org/licenses/by-nc/4.0/). 


Capsule
Summary is already known
$\begin{aligned} & \text { Secondary systemic insults after traumatic brain injury may result in fatal neurologic outcomes. Hypoxic ischemia may } \\ & \text { inflict neurologic dysfunction, ranging from mild cognitive deficits to persistent vegetative states. }\end{aligned}$
$\begin{aligned} & \text { What is new in the current study } \\ & \text { Secondary hypoxic ischemia aggravated spatial, fear, and discrimination memory deficits in an experimental traumatic } \\ & \text { brain injury model. The mechanisms underlying neurobehavioral deficits induced by secondary hypoxic ischemia fol- } \\ & \text { lowing traumatic brain injury may act partly via increased neuroinflammation and oxidative stress in the damaged ce- } \\ & \text { rebral cortex. }\end{aligned}$

\section{INTRODUCTION}

According to the World Health Organization and epidemiological data, traumatic brain injury (TBI) is a significant global public health issue and a major cause of morbidity and mortality. ${ }^{1}$ In the United States, TBI affects more than 1.5 million people annually, out of which approximately 300,000 are hospitalized and more than 50,000 deaths, accounting for approximately one-third of injuryrelated deaths. ${ }^{2,3}$

In addition to primary brain injuries, secondary insults such as hypoxia, hypotension, hypercapnia, and seizures can occur and further aggravate the injury. ${ }^{4}$ Hypoxia is a frequent secondary insult correlated with poor outcomes. ${ }^{5}$ Cerebral hypoxia has been reported in up to $44 \%$ of TBI patients with underlying brainstem injury, hypotension, impaired brain perfusion, and extracranial trauma, including airway obstruction, chest injury, and peripheral hemorrhage. ${ }^{6}$

TBI may result in various temporary or permanent physical, cognitive, behavioral, and emotional impairments. ${ }^{7}$ Among these, initial and persistent cognitive deficits are the most common, including loss of short-term memory and learning difficulties. The pathophysiology of TBI-induced neurobehavioral changes is underpinned by biochemical processes that lead to secondary brain damage, such as neurotoxic end product release, neuroinflammation, formation of reactive oxygen species (ROS), and neuronal cell death. ${ }^{4,6,8}$

Although large epidemiological studies have reported that postTBI hypoxia exacerbates neurobehavioral deficits, experimental studies using murine models examining such effects are limited. Recent studies using the Marmarou rat model of diffuse TBI revealed that secondary systemic hypoxia aggravates behavioral deficits, enhances neuroinflammation, and causes prolonged metabolic dysfunction. ${ }^{9.10}$ Murine fluid percussion and controlled cortical impact (CCI) models of focal TBI demonstrated that second- ary systemic hypoxic insults worsen functional outcomes and increase neuronal cell death. ${ }^{11}$ These post-TBI systemic hypoxia models, in which oxygen delivery is strictly controlled throughout the experimental procedure, well reflect the hypoxic conditions caused by an obstruction of the airways or other respiratory difficulties following an accident. However, there is a paucity in experimental studies combining the $\mathrm{CCl}$ mouse model with additional posttraumatic hypoxic ischemia (HI). Among TBI animal models, the $\mathrm{CCl}$ model produces the most precise injury by controlling deformation parameters, such as time, velocity, and depth of impact. Consequently, its most crucial advantages over other TBI models are the absence of risk for rebound injury as well as higher accuracy, reproducibility, and simplicity. ${ }^{12}$

Although there is limited clinical evidence of the neurological impact secondary $\mathrm{HI}$ has on $\mathrm{TBI}^{5,13}$ it is considered a critical factor that augments trauma-induced brain damage. However, the underlying mechanisms responsible for this effect remain inconclusive.

Here, we assessed the differences in neurobehavioral deficits, neuroinflammation, and oxidative stress between TBI and TBI combined with secondary $\mathrm{HI}(\mathrm{TBI}+\mathrm{HI})$ in adult male mice exposed to moderate to severe $\mathrm{CCl}$.

\section{METHODS}

\section{Animal population}

All experimental protocols were approved by the Animal Care and Use Committee at Chungnam and Chungbuk National Universities (No. 00-854-15) and adhered to the Declaration of Helsinki. A total of $88 \mathrm{C} 57 \mathrm{BI} / 6$ male mice aged 8-12 weeks were used in this study. Animals were housed in a standard 12-hour light/dark cycle with ad libitum access to food and water. The numbers of mice used were as follows: preliminary experiments to determine injury severity according to impact depth: 20; neurobehavioral 
testing: 41, of which 32 were euthanized following tiletamine/ zolazepam anesthesia on day 21 after injury; biochemical test: 27 , of which 23 were euthanized on day 3 after injury.

\section{Surgical procedures}

Mice were anesthetized with an intramuscular injection of 15 $\mathrm{mg} / \mathrm{kg}$ of tiletamine/zolazepam (Zoletil; Virbac, Carros, France). After the scalp was shaved and cleaned with betadine, a midline incision was made to expose the right parietal bone. A 4-mm-diameter circle was drawn at the center of lambda and bregma 0.5 $\mathrm{mm}$ from the midline. Right parietal craniotomy was performed using a surgical microscope and micromotor drill (Stoelting, Wood Dale, IL, USA) along the marked circle. The CCl device was calibrated with respect to the exposed dura mater within the craniotomy. Impact parameters for injury comprised a depth of $0.0,1.0$, 1.5 , and $2.0 \mathrm{~mm}$, mean velocity of $3.0 \mathrm{~m} / \mathrm{s}$, and duration of 500 $\mathrm{ms}$. Following the impact, the scalp incision was sutured with 5-0 nylon. After supine positioning we induced unilateral cerebral $\mathrm{HI}$ damage as described previously. ${ }^{14,15}$ The right carotid artery was carefully isolated without damaging the vagus nerve and double ligated with 4.0 surgical silk prior to suturing. Animals were allowed to recover with access to food and water for 2 hours before exposure to hypoxia in hypoxic chambers for 30 minutes. Oxygen levels were monitored and kept at $8 \%$, balanced with nitrogen. Chamber temperature was maintained at $35^{\circ} \mathrm{C}$. Animals were returned to their cages post procedure. Sham-operated groups underwent craniotomy without $\mathrm{CCl}$ injury. The surgical procedure is shown in Fig. 1.

\section{Health assessment score}

The mice were weighed daily. A health assessment score was recorded for each mouse daily postsurgery, ranging from $0-2,0-3$, or $0-5$ depending on the behavior assessed ( 0 indicating no deficit, 5 indicating the most severe impairment). Assessed behaviors included consciousness (0-3), interaction (0-2), ability to grasp a wire top (0-2), motor function (0-5), and activity (0-2). ${ }^{16,17}$ Sums of scores in each category yielded the overall health assessment score.

\section{Hematoxylin-eosin staining and estimation of cortical lesion volume}

Animals were anesthetized as described above and perfused with cold saline on day 21 after injury. Brains were removed, post-fixed with 10\% formalin, and paraffin embedded. Coronal sections (6$\mu \mathrm{m}$ thick) were collected at $100-\mu \mathrm{m}$ interval and stained with hematoxylin and eosin (H\&E). Micrographs of H\&E-stained sections were obtained to record and calculate the extent of injury at $1.25 \times$ magnification using a CX43 Olympus microscope (Olympus Corporation, Toyko, Japan).

Cortical lesion volumes $\left(\mathrm{mm}^{3}\right)$ were determined by calculating

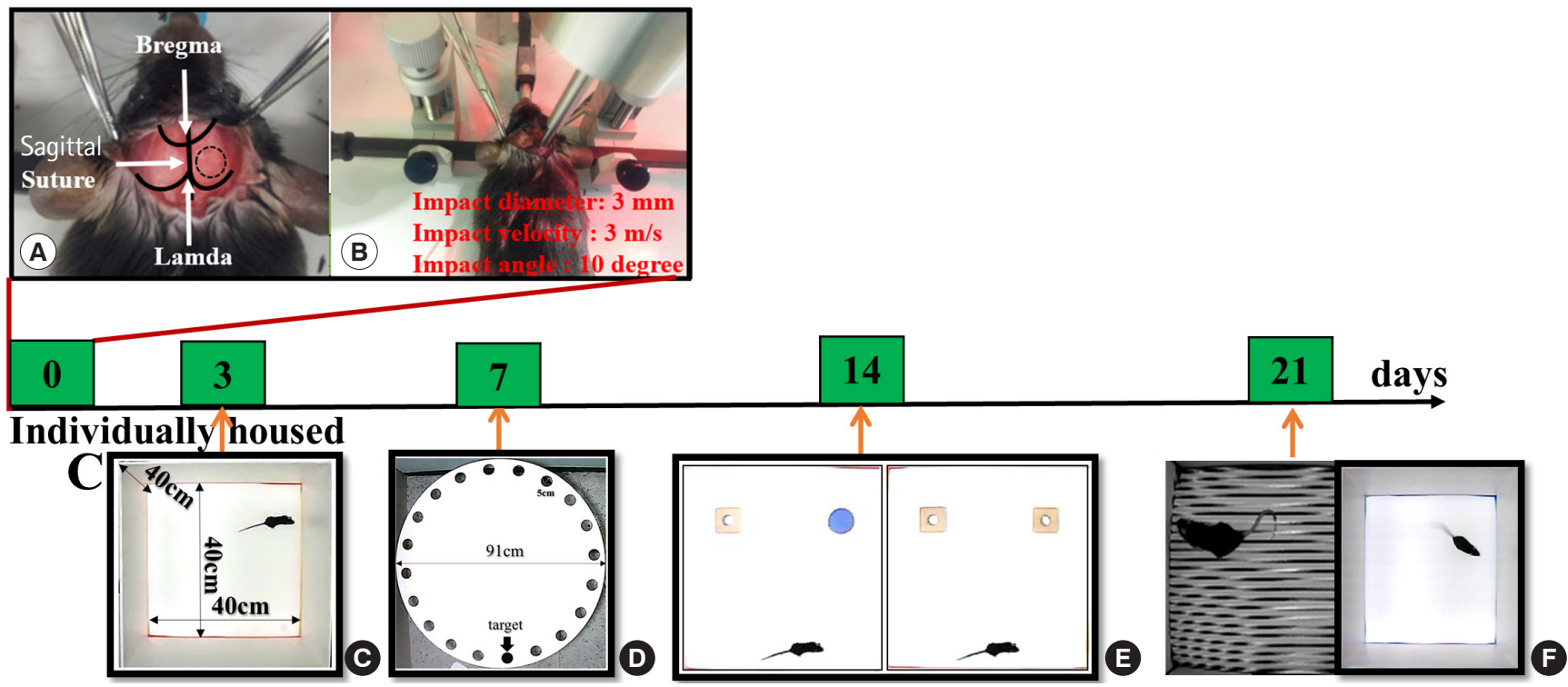

Fig. 1. Schematic timeline of experimental procedures. The procedure for controlled cortical impact is illustrated in (A) and (B). During surgery (day 0), the head of each mouse was stably fixed in a stereotactic frame using ear bars and a mouth clamp. The right skull was exposed, and a $4 \mathrm{~mm}$ circle was drawn in the center of bregma and lambda (A). The bone was removed by drilling to generate a window for impact. The impactor tip was retracted and lowered to the surface of the exposed dura until contact was made (B). Neurobehavioral tests were conducted to assess memory deficits and motor performance (C-F). Locomotor activity test (C); Barnes maze test (D); novel object recognition test (E); contextual and cued fear conditioning (F). 
the area of the lesion $\left(\mathrm{mm}^{2}\right)$ (at $1.25 \times$ magnification using the CX43 microscope) by outlining the missing cortical tissue for each section cut at 500- $\mu \mathrm{m}$ intervals and multiplying the sum of lesion areas obtained from each section with the distance between sections. Lesion volumes were analyzed using Image J 1.42 ( $\mathrm{Na}$ tional Institutes of Health, Bethesda, MD, USA).

\section{Behavioral testing}

Behavioral testing was conducted between 8 a.m. and 7 p.m. by an observer blinded to the experimental procedures. Memory was assessed using the Barnes maze test (BMT), contextual and cued fear conditioning (CCFC), and novel object recognition test (NORT) on days 7, 14, and 21 after $\mathrm{CCl}$, respectively (Fig. 1). All testing was recorded using a video tracking system with SMART ver. 3.0 (Harvard Apparatus, Holliston, MA, USA), which automatically identified post-injury behavioral changes.

\section{Locomotor activity}

Locomotor activity was measured in a white open-top acrylic box $(40 \times 40 \times 40 \mathrm{~cm})$ with an illumination intensity of 20 lux at floor level for 30 minutes. Distance moved $(\mathrm{mm})$, time spent within the center (central 25\% of box) and the area surrounding it (sec), and mean walking speed $(\mathrm{cm} / \mathrm{sec})$ were evaluated.

\section{BMT}

The BMT was conducted as previously described with minor modifications. ${ }^{18}$ The maze consisted of a white, acrylic, circular platform (91 cm diameter) with 20 equally sized holes and a black acrylic escape box $(20 \times 5 \times 6 \mathrm{~cm})$ along the perimeter. The maze was surrounded by four spatial cues at the height of the maze.

Acquisition trials. Acquisition comprised four trials per day over 3 days with 10-15 minutes inter-trial intervals. Prior to the first trial, the mice were placed in the middle of the maze in a black starting cylinder (10 cm diameter). A loud white noise (80$90 \mathrm{~dB}$ ) was sounded, inducing escape behavior. After 10 seconds, the chamber was lifted, and the mice were pretrained to enter the escape tunnel by guiding them to it and allowing them to remain there for 2 minutes. Following this training, the first trial was initiated.

At the start of each trial, the mice were placed in the black starting cylinder. Ten seconds after the onset of the noise and light, the cylinder was lifted and the animals were free to explore the maze. The trial ended when the mice entered the escape tunnel or after 3 minutes. Immediately after the mice entered the tunnel, the noise was terminated. Animals were allowed to stay within the tunnel for 1 minute. After each trial, the maze was cleaned with 70\% alcohol and rotated to eliminate intra-maze cues.
Probe trial. During the 90-second probe trial conducted on day 6 , the escape tunnel was closed. Mice were allowed to explore the maze and visit the escape tunnel. Primary latency and distance traveled to reach the tunnel were recorded.

\section{CCFC}

To assess fear learning and memory, the CCFC test was conducted as previously described with minor modifications. ${ }^{19}$ The apparatus consisted of a square chamber $(25 \times 25 \times 24 \mathrm{~cm})$ with an electrifiable grid floor, sound source, and calibrated shock generator (Panlab/Harvard Apparatus, Barcelona, Spain).

Conditioning. Animals were placed into the conditioning chamber. After 2 minutes of exploration, an 80-dB clicker (6 cps) sounded for 30 seconds to serve as a conditioned stimulus (CS) and was concomitantly terminated with a 2-second, 0.7-mA scrambled foot shock (unconditioned stimulus, US). This sequence was repeated twice, followed by a 30-second delay before mice were returned to their home cages.

Context test. After completing the conditioning session, the mice were returned to the same conditioning chamber and scored for freezing behavior to measure contextually conditioned fear. A 72-hour delay interval between the conditioning and context test was set. Mice were placed in the conditioning chamber and allowed to freely explore the chamber for 5 minutes without CS and US presentations prior to being returned to their home cages.

Cued test. The cued test was conducted on the same day as the context test. Mice were placed into a different testing chamber for 3 minutes, providing a new context unrelated to the conditioning chamber. At the end of the initial 3 minutes, a conditioned auditory cue was presented for another 3 minutes in the novel context environment. Mice were allowed to explore the chamber for 6 minutes; during the first 3 minutes, neither $\mathrm{CS}$ nor US was presented, while a CS ( $55 \mathrm{~dB}$ white noise) was presented during the final 3 minutes.

\section{NORT}

Behavior was sequentially assessed in the NORT using a 2-day protocol in the same open top acrylic box described above. ${ }^{20}$ After habituation, animals were allowed to explore two identical objects for 10 minutes (acquisition trial). The time spent exploring each object was recorded. Retention trials were performed at 1and 24-hour intervals following the acquisition trial. During retention trials, the mice were returned to the same box, in which one of the familiar objects used during acquisition was replaced by a novel object. Mice were allowed to explore freely for $10 \mathrm{~min}$ utes. Time spent exploring each object was recorded. 


\section{Measurement of oxidative stress markers}

We measured lipid peroxidation, glutathione (GSH) concentration, and superoxide dismutase (SOD) activity as oxidative stress markers on day 3 after injury. Snap-frozen cortex was homogenized with ultrasonic waves in a bead homogenizer. Cortical lipid peroxide content was determined using the modified thiobarbitu- ric acid (TBA)-reactive substances assay method as described previously. ${ }^{21}$ Briefly, an aliquot of the homogenate was added to an equal volume of $0.67 \%(\mathrm{w} / \mathrm{v}) \mathrm{TBA}$. Subsequently, $800 \mu \mathrm{L}$ of the supernatant were mixed with $100 \mu \mathrm{L}$ of $0.1 \mathrm{M}$ TBA and heated at $100^{\circ} \mathrm{C}$ for 10 minutes. After cooling, the absorbance was read at $532 \mathrm{~nm}$ using a SpectraMax 250 microplate reader (Molecular

Table 1. Physiological parameters in mice following controlled cortical impact

\begin{tabular}{|c|c|c|c|c|}
\hline & Sham & $\mathrm{HI}$ & TBI & $\mathrm{TBI}+\mathrm{HI}$ \\
\hline Number & 10 & 10 & 10 & 11 \\
\hline Age (wk) & $9.89 \pm 1.23$ & $10.01 \pm 1.20$ & $9.29 \pm 1.24$ & $9.97 \pm 1.36$ \\
\hline Body weight $(B L, g)$ & $24.02 \pm 2.45$ & $24.72 \pm 1.97$ & $24.51 \pm 4.14$ & $24.05 \pm 1.22$ \\
\hline Body temperature $\left({ }^{\circ} \mathrm{C}\right)$ & $35.64 \pm 0.50$ & $35.97 \pm 0.69$ & $35.36 \pm 0.59$ & $35.54 \pm 0.59$ \\
\hline \multicolumn{5}{|l|}{ Weight gain (\% of $\mathrm{BL}$ ) } \\
\hline POD 1 & $95.65 \pm 4.80$ & $93.10 \pm 5.30$ & $93.38 \pm 2.39$ & $89.17 \pm 4.55$ \\
\hline POD 2 & $99.20 \pm 3.24$ & $90.56 \pm 7.07$ & $94.63 \pm 3.03$ & $83.15 \pm 4.74^{*}$ \\
\hline POD 3 & $100.20 \pm 5.25$ & $94.19 \pm 7.23$ & $92.73 \pm 2.95$ & $80.45 \pm 7.99^{*}$ \\
\hline \multicolumn{5}{|l|}{ Health assessment scores } \\
\hline POD 1 & $0.17 \pm 0.41$ & $2.12 \pm 1.63$ & $0.50 \pm 0.54$ & $8.00 \pm 6.01^{* * *}$ \\
\hline POD 2 & $0.20 \pm 0.45$ & $1.36 \pm 1.22$ & $0.55 \pm 2.32$ & $5.67 \pm 4.93^{* *}$ \\
\hline POD 3 & $0.33 \pm 0.58$ & $1.04 \pm 1.65$ & $1.14 \pm 1.75$ & $10.50 \pm 6.34^{* * *}$ \\
\hline Survivals at 21 days & $10 / 10(100.0 \%)$ & $9 / 10(90.0 \%)$ & $8 / 10(80.0 \%)$ & $5 / 11(50.0 \%)^{*}$ \\
\hline
\end{tabular}

Body temperature represents the average rectal temperature during surgery.

$\mathrm{HI}$, hypoxic ischemia; $\mathrm{TBI}$, traumatic brain injury; $\mathrm{BL}$, baseline; $\mathrm{POD}$, postoperative day.

Asterisks indicate significant difference between $\mathrm{TBI}+\mathrm{HI}$ and TBI only mice. ${ }^{*} \mathrm{P}<0.05,{ }^{* *} \mathrm{P}<0.01,{ }^{* * *} \mathrm{P}<0.001$.
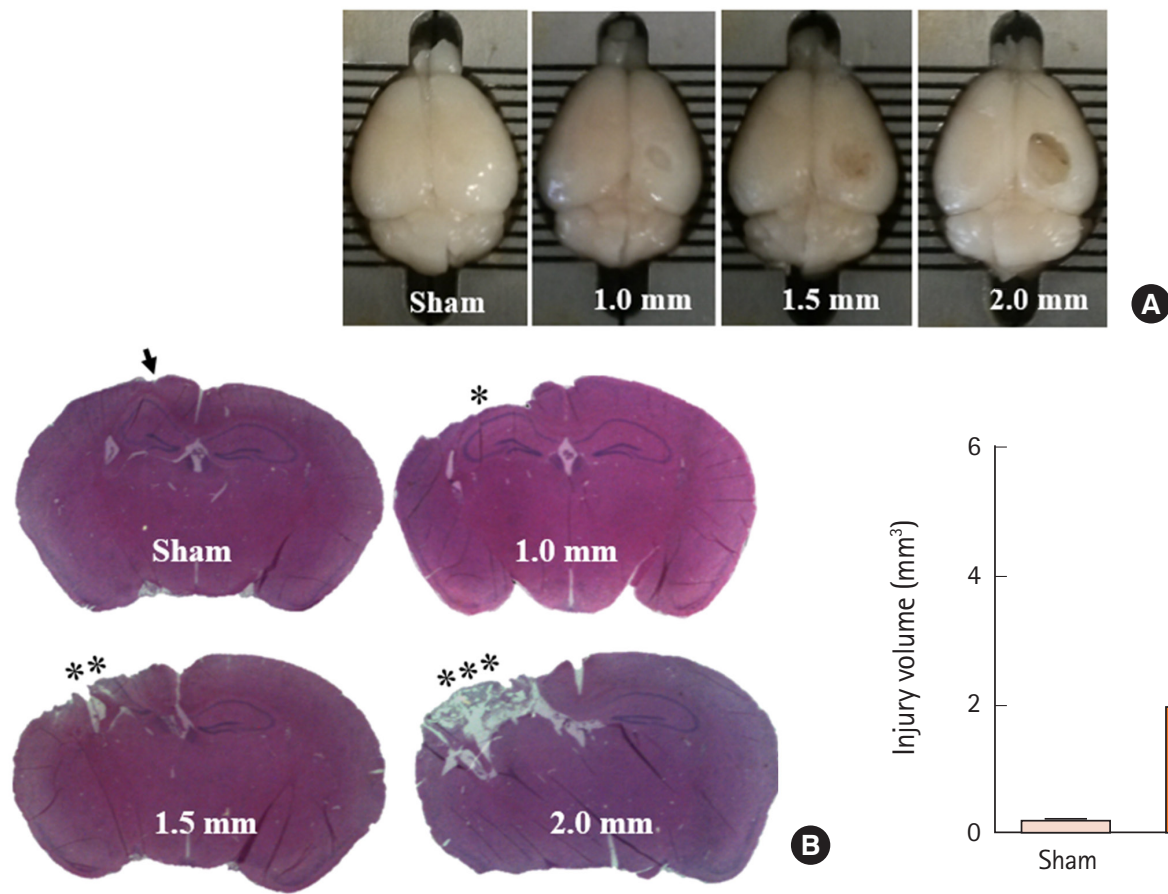

B

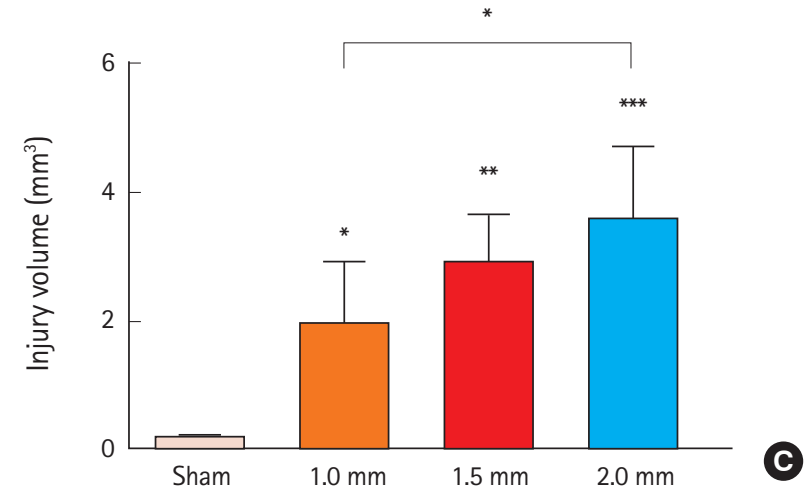

Fig. 2. Graded injury severity in adult male mice. Gross examination of the brain revealed significant graded lesions (A). Quantitative analysis of injured volume following graded controlled cortical impact showed a significant difference between injury and sham groups using one way analysis of variance with Bonferroni adjusted post hoc test $\left({ }^{*} \mathrm{P}<0.05,{ }^{* *} \mathrm{P}<0.01\right.$, $\left.{ }^{* *} \mathrm{P}<0.001\right)(\mathrm{B}, \mathrm{C})$. A significant difference was found between $1.0-\mathrm{mm}$ and $2.0-\mathrm{mm}$ depth of impact injuries $\left({ }^{*} P<0.05\right)$. The arrow indicates minimal injury from drilling. 
Devices, Sunnyvale, CA, USA). A malondialdehyde (MDA) standard was prepared from 1,1,3,3-tetraethoxypropane. GSH levels and SOD activity were determined using the GSH colorimetric assay kit (BioVision, Milpitas, CA, USA) and SOD assay kit (Cell Biolabs, $C A, U S A)$, respectively, according to the manufacturer's instructions.

\section{Measurement of proinflammatory cytokines}

On 3 day after injury, interleukin (IL)-1 $\beta$, IL-6, and tumor necrosis factor (TNF)-a levels were measured in the supernatant of homogenized brain tissue using a mouse multiplex cytokine assay kit (Multi Beads Assay BioPlex; R\&tD Systems, Minneapolis, MN, USA) according to the manufacturer's instructions. Cytokine levels were calculated according to multiplex cytokine software.

\section{Statistical analysis}

All data are presented as arithmetic mean \pm standard error of the mean. The statistical significance among groups was assessed with nonparametric Kruskal-Wallis one-way analysis of variance, followed by multiple comparisons post hoc analyses with Bonferroni methods. $\mathrm{P}<0.05$ was considered statistically significant. Data were analyzed using PASW Statistics ver. 18 (SPSS Inc., Chicago, IL, USA).
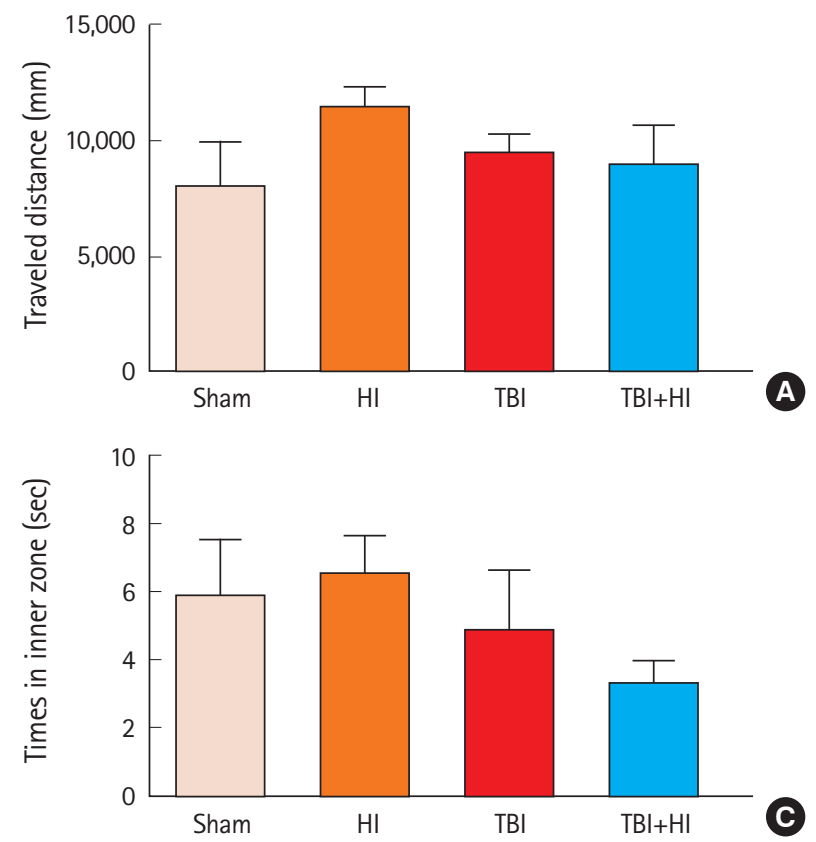

\section{RESULTS}

\section{Physiological variables}

Body weight, body temperature during surgery, age, rates of survival, and health assessment scores are shown in Table 1. Weight gain (\%) was significantly lower in the $\mathrm{TBI}+\mathrm{HI}$ group as compared to the TBI-only group on postoperative days (POD) 2-3. In contrast, health assessment scores were significantly enhanced in $\mathrm{TBI}+\mathrm{HI}$ mice as compared to TBI-only counterparts on POD 1-3. The rate of survival of $\mathrm{TBI}+\mathrm{HI}$ mice was significantly lower than that of TBI-only mice (Table 1).

\section{Cortical impact injury volumes}

To determine injury severity according to impact depth, we first impacted mouse cortices with four different depths $(0.0,1.0,1.5$, and $2.0 \mathrm{~mm}$ ) (Fig. 2A). Analysis of tissue samples collected 7 days post-injury demonstrated progressively larger brain lesions proportional to the graded depth of impact. Sham mice were not or minimally injured due to drilling. H\&E staining demonstrated that all mice exposed to 2.0-mm impact depth revealed the apparent hippocampal injury, whereas 1.0-mm impact depth did not affect hippocampi (Fig. 2B). Based on the observed differences in injury volume between tested depths of cortical impact (Fig. 2C), we applied 2.0-mm depth of impact (moderate to severe injury) to analyze the differences between TBI-only, TBI+HI, and control
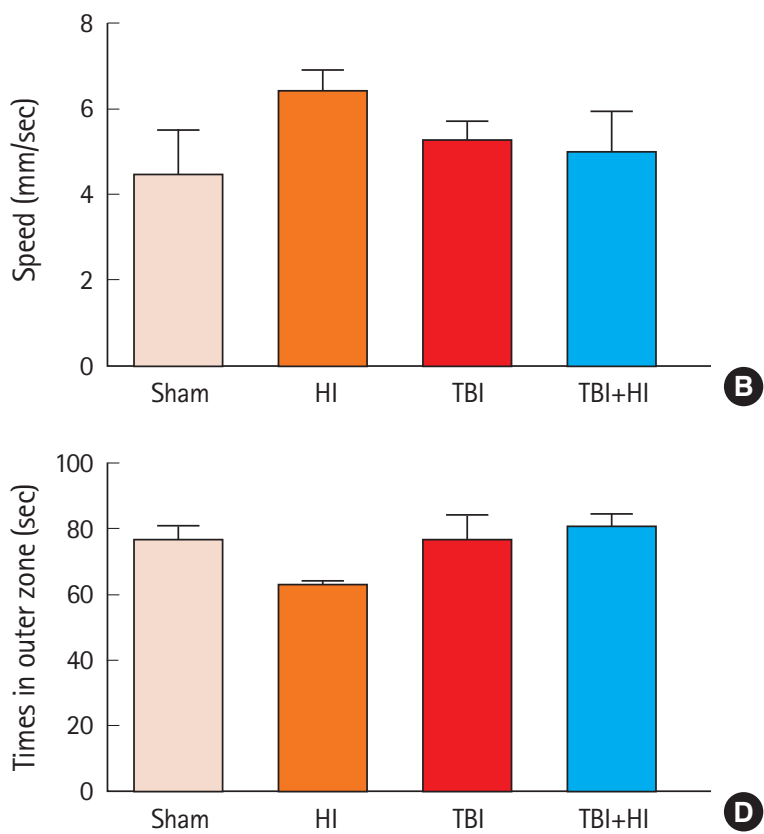

Fig. 3. Effects of traumatic brain injury (TBI) on locomotor activity in mice. Traveled distance (A); mean speed (B); time spent in the central field (C); and time spent outside the central field (D). Data were analyzed using one-way analysis of variance, followed by Bonferroni post hoc test ( $n=8-10$ per group; bars and whiskers represent mean \pm standard error of the mean). HI, hypoxic ischemia. 
groups in subsequent experiments.

\section{Neurobehavioral outcomes \\ Locomotor activity}

As shown in Fig. 3, overall locomotion was not influenced by TBI or $\mathrm{HI}$, indicating that neither significantly impacted motor behavior.

\section{Spatial learning and memory deficits in the BMT}

We next assessed spatial learning and memory using the BMT (Fig. 4A). In the acquisition phase, while there was no change in mean path length among groups (Fig. 4B), the latency to enter the escape tunnel decreased significantly over the three training days in sham mice, indicating mean performance improvement
(Fig. 4C). A significant difference was observed in the latency to enter the escape hole between $\mathrm{TBI}$ and $\mathrm{TBI}+\mathrm{HI}$, and between sham and $\mathrm{TBI}+\mathrm{HI}$ mice (Fig. 4C). No significant difference was observed in average speed among groups (Fig. 4D). In the retention phase, no significant differences were observed in primary path length to the target hole among groups (Fig. 4E). In contrast, an unpaired t-test revealed significant differences in primary latency to enter the target hole and adjacent holes between $\mathrm{TBI}$ and $\mathrm{TBI}+\mathrm{HI}$ mice, and between sham and TBI mice (Fig. 4F). These findings indicate that $\mathrm{TBI}$ caused retention memory deficits that were exacerbated by $\mathrm{HI}$ (Fig. 4F).

\section{CCFC}

Acquisition of cued fear was assessed as locomotor activity chang-
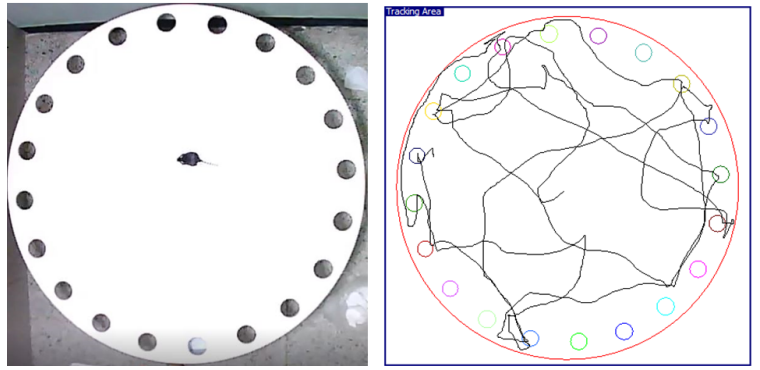

A
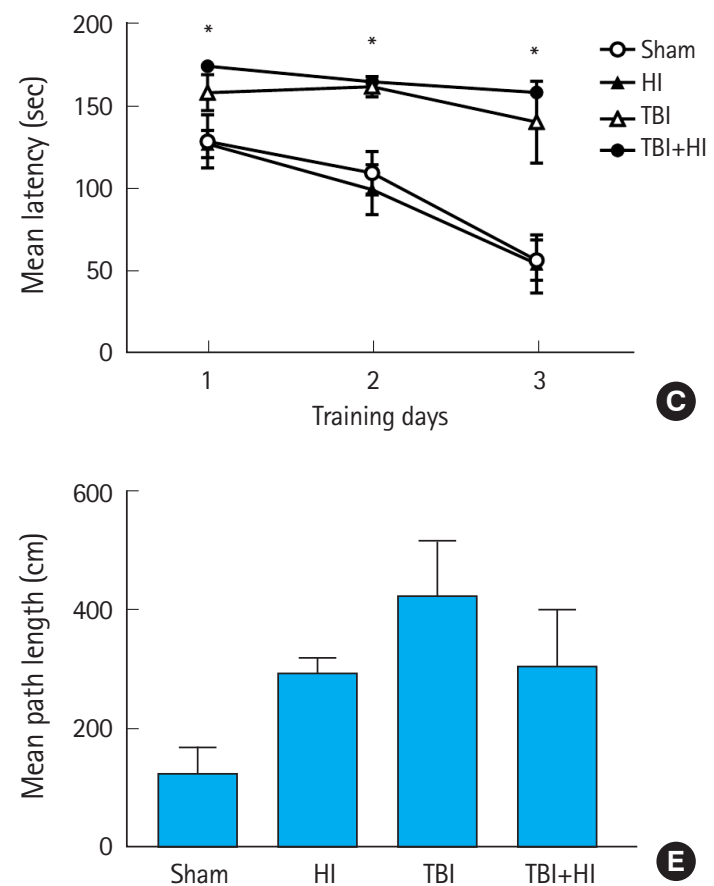
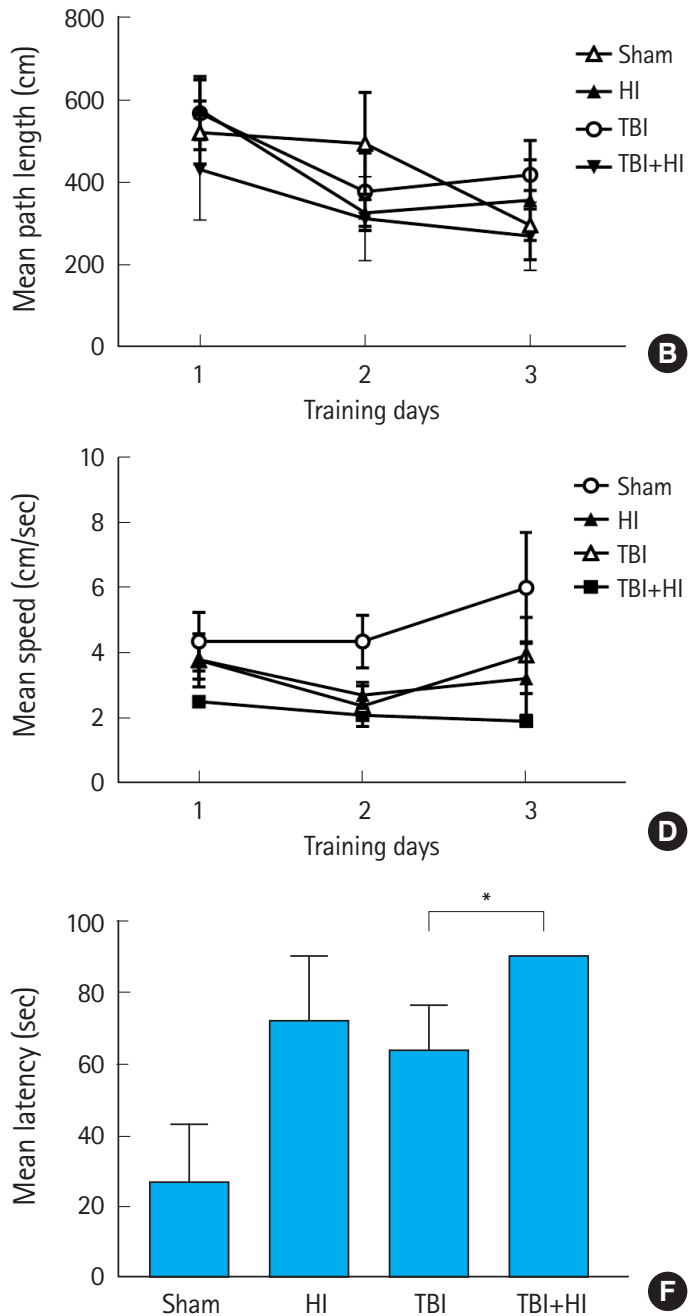

Fig. 4. Spatial learning and memory following traumatic brain injury (TBI). The Barnes maze was used to determine latencies, distances, errors, and speed to find the escape box. Tracking plots of a single mouse exploring the Barnes maze (A); mean path length in acquisition trials (B); mean latency in acquisition trials (C); mean speed in acquisition trials (D); mean path length on day 5 of retention trials $(\mathrm{E})$; mean latency in retention trials (F). Asterisks indicate significant differences; ${ }^{*} P<0.05$ ( $n=6-8$ per group; bars and whiskers represent mean \pm standard error of the mean). HI, hypoxic ischemia. 

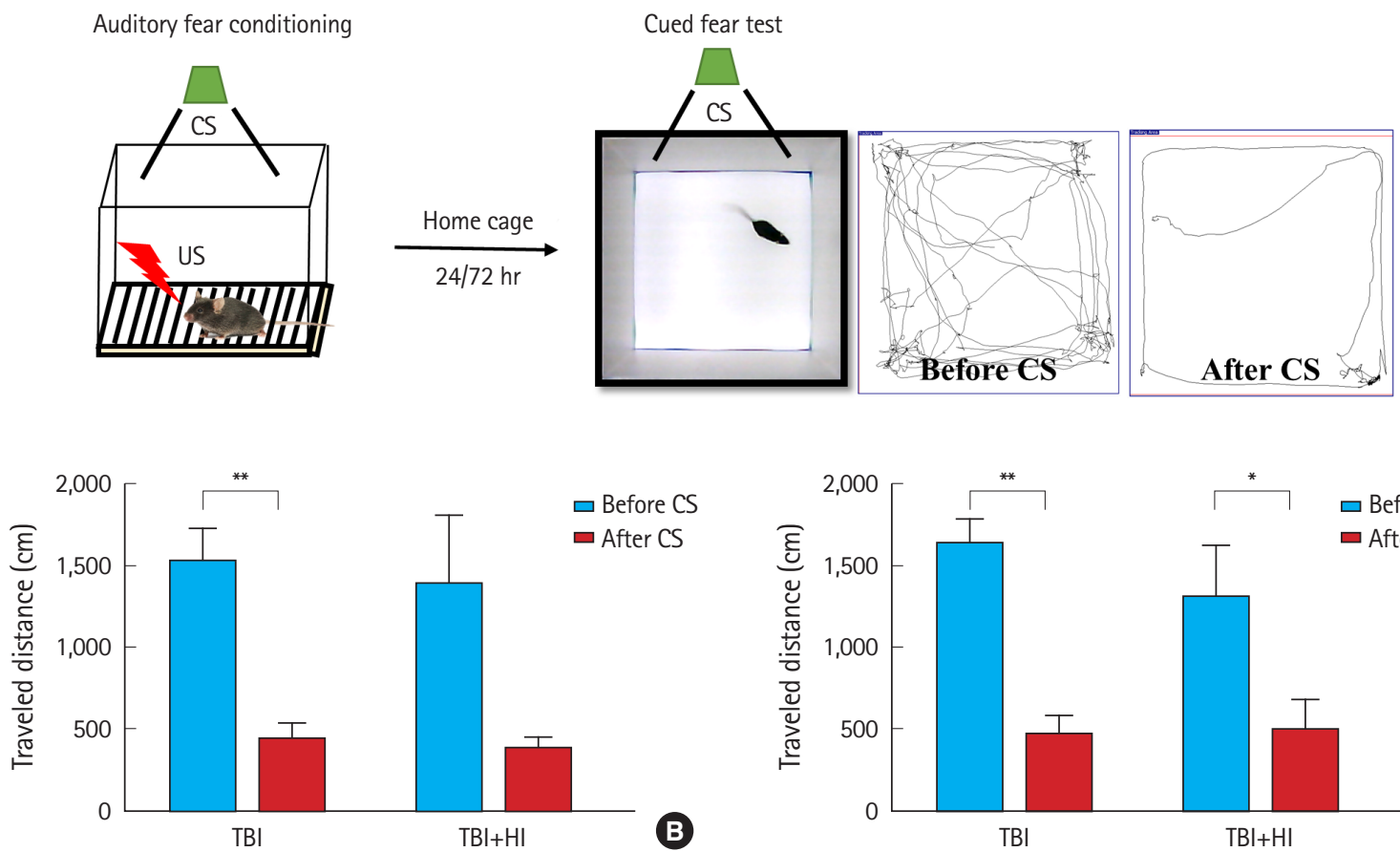

TBI

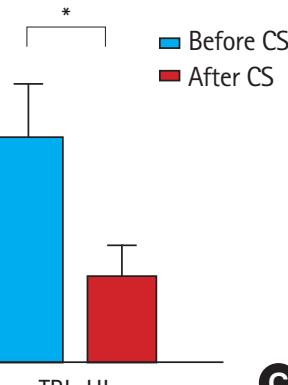

Fig. 5. Impaired fear recall in traumatic brain injury (TBI)+hypoxic ischemia (HI) mice following contextual fear conditioning. Experimental design and fear conditioning procedure (A). Cued fear recall on day 1 (B) and day 3 (C). TBI+HI mice demonstrated deficits in cued fear recall relative to TBI-only mice. Asterisks indicate significant differences; ${ }^{*}<<0.05,{ }^{* *} \mathrm{P}<0.01(\mathrm{n}=6-8$ per group; bars and whiskers represent mean \pm standard error of the mean). $C S$, conditioned stimulus (white noise, $55 \mathrm{~dB}$ ); US, unconditioned stimulus (0.3-mA electric foot shock; 2 seconds).

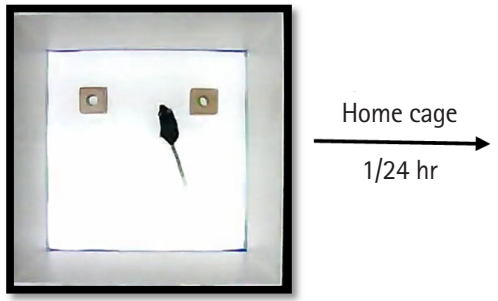

Training day

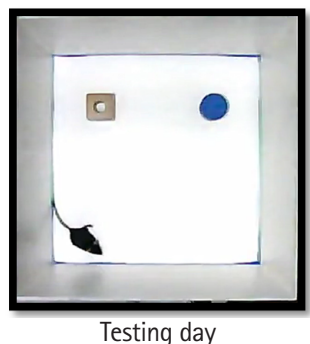

Testing day

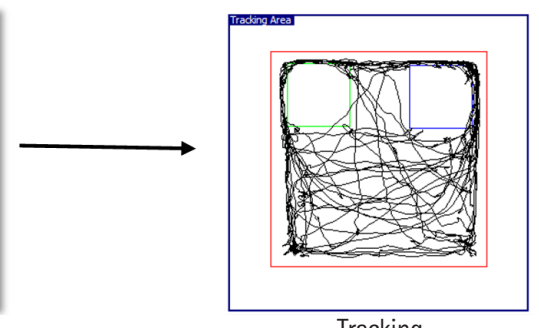

Tracking
A
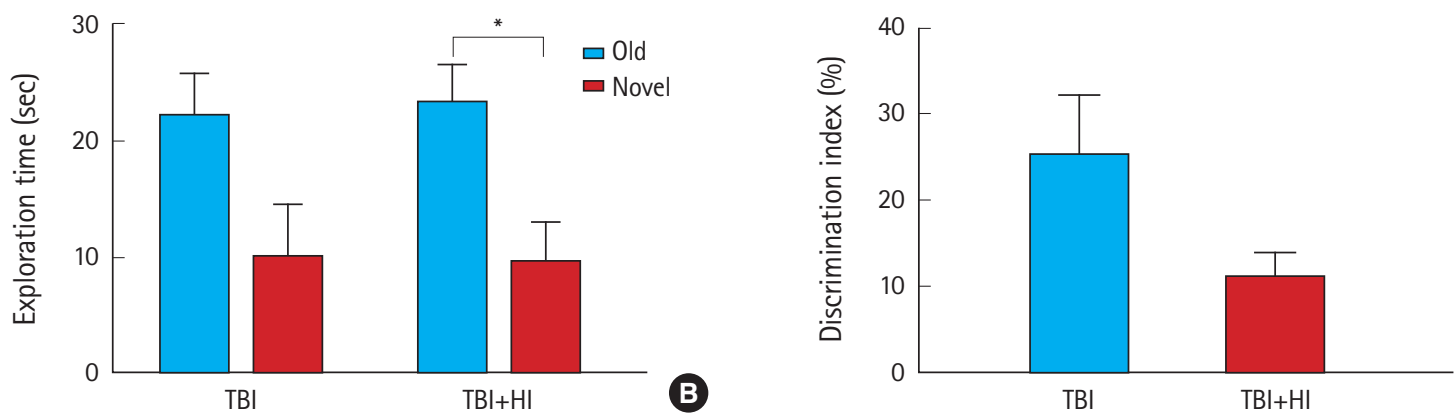

C

Fig. 6. Impaired discrimination recall in traumatic brain injury (TBI)+hypoxic ischemia (HI) mice in the novel object recognition test. Experimental design and representative tracking plot (A). Locomotor activity was recorded by a video tracking system in the testing phase. TBI+HI mice spent less time exploring the novel object, whereas TBI-only mice did not exhibit a preference for the novel object (B). Novel object discrimination index percentage between TBI-only and $\mathrm{TBI}+\mathrm{HI}$ mice, indicating impaired cognitive ability in $\mathrm{TBI}+\mathrm{HI}$ compared to TBI-only mice (C). Asterisks indicate significant differences; ${ }^{*} \mathrm{P}<0.05$ ( $n=6-8$ per group; bars and whiskers represent mean \pm standard error of the mean). 
es due to freezing during tone presentation following foot shock (Fig. 5A). A significant difference was observed in mean changes in locomotion during pre-cue and cue presentation between TBIonly and $\mathrm{TBI}+\mathrm{HI}$ groups on days 1 (Fig. 5B) and 3 (Fig. 5C) after fear conditioning. TBI-only mice demonstrated good learning ability, as they avoided entering the chamber in which they received a foot shock on days 1 and 3. No significant changes in locomotion were observed in $\mathrm{TBI}+\mathrm{HI}$ mice during pre-cue and cue presentation on day 1 , indicating deficits in cued fear recall relative to TBI-only mice.

\section{NORT}

Potential changes in cognitive function caused by $\mathrm{HI}$ were assessed using the NORT (Fig. 6A). TBI+HI mice displayed significantly lower preference for the object moved to a novel place compared to the object that remained in the same (familiar) place; these mice spent $9.5 \%$ of object exploration time in the test phase with the object that was moved to a novel place (Fig. 6B). No significant difference in exploration time was observed in TBI-only mice between familiar and novel objects (Fig. 6B). Comparison of the novel object discrimination index percentage between TBI-only and
$\mathrm{TBI}+\mathrm{HI}$ mice revealed a trend towards impaired cognitive ability in $\mathrm{TBI}+\mathrm{HI}$ mice (Fig. $6 \mathrm{C}$ ).

\section{Oxidative stress following TBI}

We measured the lipid peroxidation marker MDA (Fig. 7A), GSH levels as an indicator for glutathione peroxidase (GPx) activity (Fig. 7B), and SOD activity (Fig. 7C) as markers of oxidative stress, and compared the levels of these markers among groups 72 hours following surgery. MDA levels were significantly lower in $\mathrm{TBI}+\mathrm{HI}$ mice than in TBI-only mice (Fig. 7A). GPx activity was significantly higher in $\mathrm{TBI}+\mathrm{HI}$ mice than in sham counterparts $(\mathrm{P}<0.05)$ (Fig. 7B). No significant differences in tissue SOD levels were observed among groups (Fig. 7C).

\section{Proinflammatory cytokine expression after TBI}

We compared the concentrations (pg/mg protein) of cytokines IL-6 (Fig. 8A), IL-1 $\beta$ (Fig. 8B), and TNF-a (Fig. 8C) among groups 72 hours postsurgery. IL-6 levels were significantly higher in $\mathrm{TBI}+\mathrm{HI}$ mice compared to TBI-only and sham mice. We found no significant differences in tissue IL-1 $\beta$ and TNF- $a$ levels between groups (Fig. 8B, C).
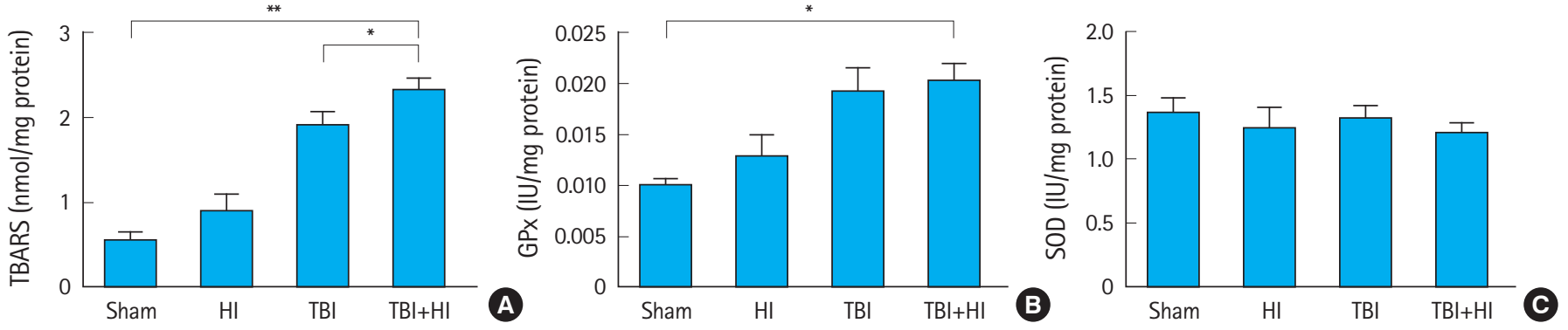

Fig. 7. Oxidative stress following traumatic brain injury (TBI). Hypoxic ischemia (HI) following TBI increased thiobarbituric acid reactive substance (TBARS) levels in traumatized brain hemispheres (A). In $\mathrm{TBI}+\mathrm{HI}$ mice, glutathione peroxidase (GPx) activity was significantly increased in the traumatized brain hemispheres compared to sham mice (B). TBI or HI did not affect superoxide dismutase (SOD) activity in the traumatized hemispheres (C). Asterisks indicate significant differences; ${ }^{*} \mathrm{P}<0.05,{ }^{* *} \mathrm{P}<0.01$ ( $\mathrm{n}=5-7$ per group; bars and whiskers represent mean \pm standard error of the mean). Data were analyzed using one-way analysis of variance with Kruskal-Wallis test.
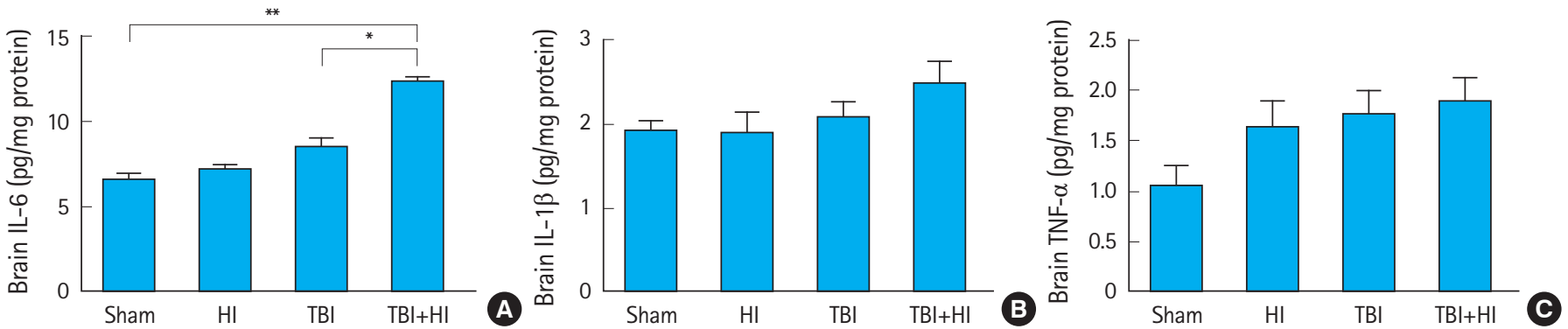

Fig. 8. Interleukin-6 levels were increased in mice after traumatic brain injury (TBI) with additional hypoxic ischemia (HI). The concentrations (pg/mg protein) of cytokines interleukin (IL)-6 (A), IL-1 $\beta$ (B), and tumor necrosis factor (TNF)- $\alpha$ (C) were measured in traumatized hemisphere homogenates of sham, $\mathrm{HI}, \mathrm{TBI}-$ only, and $\mathrm{TBI}+\mathrm{HI}$ mice 72 hours post-injury. Asterisks indicate significant differences; ${ }^{*} \mathrm{P}<0.05,{ }^{* *} \mathrm{P}<0.01$ ( $\mathrm{n}=5-7$ per group; bars and whiskers represent mean \pm standard error of the mean). Data were analyzed using one-way analysis of variance with Kruskal-Wallis test. 


\section{DISCUSSION}

$\mathrm{HI}$ can inflict severe neurologic disability across all age groups. Birth asphyxia is the leading cause of neonatal encephalopathy, and cardiac arrest is the main cause of $\mathrm{HI}$-mediated brain injury with subsequent morbidity and mortality. ${ }^{22,23}$ The pathophysiology of this injury encompasses a heterogeneous cascade induced by the immediate cessation of cerebral blood flow ${ }_{1}^{24}$ resulting in cellular energy failure, excitotoxicity, generation of free radicals, and apoptosis. ${ }^{22,25}$

Systemic hypoxia following TBI simulates clinical situations of purely hypoxic insults such as airway obstruction, asphyxiation, and carbon monoxide poisoning, which exacerbate neurological deficits and increase lesion size, brain edema, and neuronal death. ${ }^{26-28}$ However, the effects of post-traumatic $\mathrm{HI}$ after focal brain injury have not been addressed. We thus explored the impact of HI using a model of focal $\mathrm{CCl}$ followed by carotid occlusion alongside 60-min hypoxic ventilation.

Our results demonstrate that mice exposed to $\mathrm{CCl}$ and $\mathrm{HI}$ had a higher mortality rate and reduction in body weight than $\mathrm{CCl}-$ only mice. In addition, compared to $\mathrm{CCl}$ alone, post-traumatic $\mathrm{HI}$ increased neurobehavioral deficits in the BMT, CCFC, and NORT. These data resemble clinical findings in TBI patients, indicating that post-traumatic HI worsens neurological outcomes and prolongs recovery. ${ }^{5}$ Our findings also highlight that a post-traumatic hypoxic insult has persistent detrimental effects on inflammatory responses and oxidative stress.

Cognitive deficits resulting from TBI include impaired memory and learning, attention, concentration, processing speed, executive functioning, and word-finding difficulties. ${ }^{29}$ Factors contributing to TBI-induced memory impairments include alterations in neural circuits associated with memory function. ${ }^{30}$ We previously reported that $2.0-\mathrm{mm} \mathrm{CCl}$ injury resulted in significant spatial learning and memory deficits when compared to sham animals. ${ }^{31}$ Here, we employed three behavioral paradigms to assess learning and memory over 3 weeks. The BMT is an efficient cognitive task test to assess spatial/non-spatial learning following $\mathrm{CCl}$ injury in adult mice. ${ }^{31}$ Compared to $\mathrm{CCl}$ alone, additional $\mathrm{HI}$ aggravated spatial learning and memory. Hippocampal lesions have deleterious effects on the ability to discriminate fear-associated contexts from a neutral environment. ${ }^{32} \mathrm{~A}$ recent study reported that $\mathrm{CCl}$ does not affect the neural mechanisms of fear and extinction within the first 2 weeks of injury. ${ }^{33}$ Using CCFC, we here demonstrate that compared to $\mathrm{CCl}$ alone, additional $\mathrm{HI}$ impaired fear-associated learning and memory. Unlike TBI-only animals, $\mathrm{TBI}+\mathrm{HI}$ mice displayed significantly lower preference for the object moved to a novel place compared to the familiar one in the NORT, suggesting that $\mathrm{HI}$ affects memory function. ${ }^{34}$ It is known that recognition memory is impaired in humans and animals with TBI. ${ }^{35,36}$ Our results provide evidence that an additional secondary insult, such as $\mathrm{HI}$, may further exacerbate TBI-inflicted damage.

$\mathrm{HI}$ injury to the mammalian brain can induce neurodegenerative responses. Unilateral HI mice exhibit unilateral hippocampal, cortical, and striatal tissue loss, and decreased density of hippocampal CA1 neurons. The hippocampus is susceptible to $\mathrm{HI}$ injury, and loss of hippocampal neurons may underlie $\mathrm{HI}$-associated learning and memory deficits. ${ }^{37,38}$ In our study, HI-only and sham mice showed comparable performance in the BMT, suggesting intact hippocampal function. This implies that $\mathrm{HI}$ injury through onevessel occlusion combined with 1-hour hypoxia in adult mice is sublethal. In contrast, we observed that $\mathrm{HI}$ induced significant learning and memory deficits following TBI.

Neuroinflammation is a prominent short-and long-term consequence of neuronal injury after TBI. It involves the activation of microglia and astrocytes, which release inflammatory mediators within the brain, and the subsequent recruitment of peripheral immune cells. ${ }^{39}$ Animal models of TBI have been developed and proven valuable to elucidate the pathophysiology of the injury and enable preclinical assessment of the safety and efficacy of novel therapies. ${ }^{39}$ Based on such models, it was shown that TBI induces a robust elevation of cytokines in the brain. ${ }^{40}$ Our data did not reveal significant changes in brain cytokine production in TBI-only mice compared to sham controls 3 days after injury. In contrast, whilst IL-1 $\beta$ and TNF- $\alpha$ production remained unchanged, we observed a significant increase in IL-6 levels within $\mathrm{TBI}+\mathrm{HI}$ brains compared to TBI-only and sham counterparts. IL-6 is involved in various physiological and pathophysiological processes, such as regulation of inflammation, immunity, bone metabolism, hematopoiesis, and neural development. IL-6 mRNA is upregulated within minutes of TBI, and increased IL-6 protein levels are detectable within an hour. ${ }^{40}$ In this study, IL-6 levels were not different in TBI-only mice compared to sham mice, but were significantly increased in $\mathrm{TBI}+\mathrm{HI}$ animals compared to TBI-only and sham counterparts 3 days following the injury. These results suggest that additional $\mathrm{HI}$ prolonged neuroinflammation, which may have already been cleared in TBI-only animals.

The intracellular antioxidant defense mechanism increases as an adaptive response to oxidative stress. Upon TBI, various oxidative stress markers such as lipid peroxides are produced in the brain, while antioxidant defense enzymes such as GPx and SOD are decreased in their activities; this imbalance is directly related to TBI pathogenesis. ${ }^{41} \mathrm{GSH}$ is a major thiol tripeptide that plays a crucial role in the cellular antioxidant defense system by scavenging free radicals and other ROS, removing hydrogen and lipid per- 
oxides, and preventing oxidation of biomolecules ${ }_{i}^{40}$ we here measured GSH levels as an indicator for GPx activity. In the adult murine brain after $\mathrm{TBI}, \mathrm{GPx}$ activity is increased within 24 hours after trauma. ${ }^{42}$ In this study, we did not observe an increase in GPx levels 72 hours after TBI. However, when TBI was followed by HI, the activity of this enzyme was significantly elevated compared to sham mice, suggesting that $\mathrm{HI}$ exacerbated oxidative stress. Further studies are needed to examine time-dependent changes in oxidative stress markers. SOD is a crucial enzyme in cellular antioxidant systems that plays critical roles in the elimination of excess ROS in living organisms. It converts superoxide radicals to hydrogen peroxide, which is subsequently converted to water by catalase and GPx. In contrast to the change observed in GPx activity, SOD remained unchanged following TBI/HI.

MDA is an end-product formed during lipid peroxidation. A rise in its levels reflects an imbalance between endogenous antioxidants and oxidative stress. Elevated levels of MDA can be found in patients with TBI compared to controls, and MDA concentrations correlate with mortality following severe $\mathrm{TB} I{ }^{43}$ In this study, we observed a significant increase in this lipid peroxidation marker in $\mathrm{TBI}+\mathrm{HI}$ mice compared to sham and TBI-only animals 72 hours post-injury. Taken together, our findings suggest that posttraumatic HI significantly aggravated oxidative stress.

Overall, our study provides potential mechanisms underlying neurobehavioral outcomes following secondary $\mathrm{HI}$ insult after TBI. Thus, the prolonged neuroinflammation and oxidative stress in hippocampal neurons caused by $\mathrm{HI}$ resulted in aggravated spatial, fear, and discrimination memory deficits.

This study has several limitations. First, we used the one-vessel hypoxia model for $\mathrm{HI}$, which does not take into account cardiac arrest. Second, we only impacted the focal right parietal lobe; further investigation of other types of focal brain and diffuse brain injuries is necessary. Finally, we did not administer impacts beyond $2.0 \mathrm{~mm}$ ( 2.5 or $3.0 \mathrm{~mm}$ ) to avoid high mortality rates after surgery.

In summary, we provide evidence that secondary $\mathrm{HI}$ insult augmented spatial, fear, and discrimination memory deficits in an experimental TBI model. Results from this study suggest that TBI+HImediated neurobehavioral deficits may be at least partly explained by increased neuroinflammation and oxidative stress. Understanding these effects is an important first step in elucidating $\mathrm{HI}$-associated pathophysiological progression and the foundation for developing appropriate therapeutics for post-traumatic $\mathrm{HI}$.

\section{CONFLICT OF INTEREST}

No potential conflict of interest relevant to this article was reported.

\section{ACKNOWLEDGMENTS}

This research was supported by the Basic Science Research Program through the National Research Foundation of Korea funded by the Ministry of Education, Science and Technology (2015R1A 2A2A01002303 and NRF-2016R1D1A1B04930247).

\section{REFERENCES}

1. Popescu C, Anghelescu A, Daia C, Onose G. Actual data on epidemiological evolution and prevention endeavours regarding traumatic brain injury. J Med Life 2015;8:272-7.

2. Coronado VG, Xu L, Basavaraju SV, et al. Surveillance for traumatic brain injury-related deaths: United States, 1997-2007. MMWR Surveill Summ 2011;60:1-32.

3. Centers for Disease Control and Prevention (CDC). CDC grand rounds: reducing severe traumatic brain injury in the United States. MMWR Morb Mortal Wkly Rep 2013;62:549-52.

4. Bellander BM, Olafsson IH, Ghatan PH, et al. Secondary insults following traumatic brain injury enhance complement activation in the human brain and release of the tissue damage marker S100B. Acta Neurochir (Wien) 2011;153:90-100.

5. Chi JH, Knudson MM, Vassar MJ, et al. Prehospital hypoxia affects outcome in patients with traumatic brain injury: a prospective multicenter study. J Trauma 2006;61:1134-41.

6. Yan EB, Satgunaseelan L, Paul E, et al. Post-traumatic hypoxia is associated with prolonged cerebral cytokine production, higher serum biomarker levels, and poor outcome in patients with severe traumatic brain injury. J Neurotrauma 2014;31: 618-29.

7. Castano Monsalve $B$, Laxe $S$, Bernabeu Guitart M, Vilarrasa $A B$, Quemada Jl. Behavioral scales used in severe and moderate traumatic brain injury. NeuroRehabilitation 2014;35:6776.

8. Prins $M$, Greco T, Alexander D, Giza CC. The pathophysiology of traumatic brain injury at a glance. Dis Model Mech 2013; 6:1307-15.

9. Yan EB, Hellewell SC, Bellander BM, Agyapomaa DA, Morganti-Kossmann MC. Post-traumatic hypoxia exacerbates neurological deficit, neuroinflammation and cerebral metabolism in rats with diffuse traumatic brain injury. J Neuroinflammation $2011 ; 8: 147$.

10. Hellewell SC, Yan EB, Agyapomaa DA, Bye N, Morganti-Kossmann MC. Post-traumatic hypoxia exacerbates brain tissue damage: analysis of axonal injury and glial responses. J Neurotrauma 2010;27:1997-2010.

11. Thelin EP. Experimental models combining traumatic brain 
injury and hypoxia. Methods Mol Biol 2016;1462:459-79.

12. Xiong Y, Mahmood A, Chopp M. Animal models of traumatic brain injury. Nat Rev Neurosci 2013;14:128-42.

13. Widdel L, Winston KR. Prognosis for children in cardiac arrest shortly after blunt cranial trauma. J Trauma 2010;69:783-8.

14. Pichiule P, Chavez JC, Schmidt AM, Vannucci SJ. Hypoxia-inducible factor-1 mediates neuronal expression of the receptor for advanced glycation end products following hypoxia/ischemia. J Biol Chem 2007;282:36330-40.

15. Gilbert RW, Costain WJ, Blanchard ME, Mullen KL, Currie RW, Robertson HA. DNA microarray analysis of hippocampal gene expression measured twelve hours after hypoxia-ischemia in the mouse. J Cereb Blood Flow Metab 2003;23:1195-211.

16. Kosaka Y, Quillinan N, Bond C, Traystman R, Hurn P, Herson P. GPER1/GPR30 activation improves neuronal survival following global cerebral ischemia induced by cardiac arrest in mice. Transl Stroke Res 2012;3:500-7.

17. Allen $D$, Nakayama $S$, Kuroiwa M, et al. SK2 channels are neuroprotective for ischemia-induced neuronal cell death. J Cereb Blood Flow Metab 2011;31:2302-12.

18. Patil SS, Sunyer B, Hoger $H$, Lubec G. Evaluation of spatial memory of $\mathrm{C} 57 \mathrm{BL} / 6 \mathrm{~J}$ and $\mathrm{CD} 1$ mice in the Barnes maze, the Multiple T-maze and in the Morris water maze. Behav Brain Res 2009;198:58-68.

19. Shoji H, Takao K, Hattori S, Miyakawa T. Contextual and cued fear conditioning test using a video analyzing system in mice. J Vis Exp 2014:50871.

20. Barnes SA, Pinto-Duarte A, Kappe $A$, et al. Disruption of mGluR5 in parvalbumin-positive interneurons induces core features of neurodevelopmental disorders. Mol Psychiatry 2015;20:116172.

21. Dawn-Linsley M, Ekinci FJ, Ortiz D, Rogers E, Shea TB. Monitoring thiobarbituric acid-reactive substances (TBARs) as an assay for oxidative damage in neuronal cultures and central nervous system. J Neurosci Methods 2005;141:219-22.

22. Zhu $X$, Yan J, Bregere $C$, et al. RBM3 promotes neurogenesis in a niche-dependent manner via IMP2-IGF2 signaling pathway after hypoxic-ischemic brain injury. Nat Commun 2019; 10:3983.

23. Heinz UE, Rollnik JD. Outcome and prognosis of hypoxic brain damage patients undergoing neurological early rehabilitation. BMC Res Notes 2015;8:243.

24. Sekhon MS, Ainslie PN, Griesdale DE. Clinical pathophysiology of hypoxic ischemic brain injury after cardiac arrest: a "twohit" model. Crit Care 2017;21:90.

25. Busl KM, Greer DM. Hypoxic-ischemic brain injury: pathophysiology, neuropathology and mechanisms. NeuroRehabili- tation 2010;26:5-13.

26. Clark RS, Kochanek PM, Dixon CE, et al. Early neuropathologic effects of mild or moderate hypoxemia after controlled cortical impact injury in rats. J Neurotrauma 1997;14:179-89.

27. Matsushita Y, Bramlett HM, Alonso O, Dietrich WD. Posttraumatic hypothermia is neuroprotective in a model of traumatic brain injury complicated by a secondary hypoxic insult. Crit Care Med 2001;29:2060-6.

28. Van Putten HP, Bouwhuis MG, Muizelaar JP, Lyeth BG, Berman RF. Diffusion-weighted imaging of edema following traumatic brain injury in rats: effects of secondary hypoxia. J Neurotrauma 2005;22:857-72.

29. Ashman TA, Gordon WA, Cantor JB, Hibbard MR. Neurobehavioral consequences of traumatic brain injury. Mt Sinai J Med 2006;73:999-1005.

30. Paterno R, Folweiler KA, Cohen AS. Pathophysiology and treatment of memory dysfunction after traumatic brain injury. Curr Neurol Neurosci Rep 2017;17:52.

31. Lee SW, Jang MS, Jeong SH, Kim H. Exploratory, cognitive, and depressive-like behaviors in adult and pediatric mice exposed to controlled cortical impact. Clin Exp Emerg Med 2019; 6:125-37.

32. Balogh SA, Radcliffe RA, Logue SF, Wehner JM. Contextual and cued fear conditioning in $\mathrm{C} 57 \mathrm{BL} / 6 \mathrm{~J}$ and $\mathrm{DBA} / 2 \mathrm{~J}$ mice: context discrimination and the effects of retention interval. Behav Neurosci 2002;116:947-57.

33. Sierra-Mercado D, McAllister LM, Lee CC, Milad MR, Eskandar EN, Whalen MJ. Controlled cortical impact before or after fear conditioning does not affect fear extinction in mice. Brain Res 2015;1606:133-41.

34. Winters BD, Saksida LM, Bussey TJ. Object recognition memory: neurobiological mechanisms of encoding, consolidation and retrieval. Neurosci Biobehav Rev 2008;32:1055-70.

35. Chuah YM, Maybery MT, Fox AM. The long-term effects of mild head injury on short-term memory for visual form, spatial location, and their conjunction in well-functioning university students. Brain Cogn 2004;56:304-12.

36. Rachmany L, Tweedie D, Rubovitch V, et al. Cognitive impairments accompanying rodent mild traumatic brain injury involve p53-dependent neuronal cell death and are ameliorated by the tetrahydrobenzothiazole PFT-a. PLoS One 2013;8:e79837.

37. Fugate JE, Moore SA, Knopman DS, et al. Cognitive outcomes of patients undergoing therapeutic hypothermia after cardiac arrest. Neurology 2013;81:40-5.

38. Wilder Schaaf KP, Artman LK, Peberdy MA, et al. Anxiety, depression, and PTSD following cardiac arrest: a systematic review of the literature. Resuscitation 2013;84:873-7. 
39. Chiu CC, Liao YE, Yang LY, et al. Neuroinflammation in animal models of traumatic brain injury. J Neurosci Methods 2016; 272:38-49.

40. Woodcock T, Morganti-Kossmann MC. The role of markers of inflammation in traumatic brain injury. Front Neurol 2013; 4:18.

41. Rodriguez-Rodriguez A, Egea-Guerrero JJ, Murillo-Cabezas F, Carrillo-Vico A. Oxidative stress in traumatic brain injury. Curr
Med Chem 2014;21:1201-11.

42. Ozdemir D, Uysal N, Gonenc S, et al. Effect of melatonin on brain oxidative damage induced by traumatic brain injury in immature rats. Physiol Res 2005;54:631-7.

43. Lorente L, Martin MM, Abreu-Gonzalez P, et al. Association between serum malondialdehyde levels and mortality in patients with severe brain trauma injury. J Neurotrauma 2015; 32:1-6. 